\title{
Prevalence of pre-diabetes/type 2 diabetes among adolescents (10-19 years) and its association with different measures of overweight/obesity in India: a gendered perspective
}

\author{
Pradeep Kumar $^{1}$ (D), Shobhit Srivastava ${ }^{1}$ (D), Prem Shankar Mishra ${ }^{2^{*}}$ (D) and E. T. Krishnan Mooss ${ }^{3}$
}

\begin{abstract}
Background: The International Diabetes Federation (IDF) estimated that 1.1 million children and adolescents aged 14-19years are living with diabetes. Diabetes is a chronic, progressive disease characterized by elevated levels of blood glucose. It is also recognized as a complex disease that affects people of different ages due to different causes. The present study aims to estimate the prevalence of pre-diabetes/diabetes at the national level. Additionally, the respective study determines the factors associated with pre-diabetes/diabetes conditions among adolescents at the national level.

Methods: The data for this study was carried out from the Comprehensive National Nutrition Survey (CNNS), the first-ever nationally representative nutrition survey of children and adolescents in India. The study used a sample size of 17,865 adolescent boys and 17,965 adolescent girls for the analysis. Descriptive statistics, bivariate analysis, and logistic regression analysis were done to carve out the results.

Results: The prevalence of pre-diabetes/diabetes was 12.3\% and $8.4 \%$ among adolescent boys and girls in India, respectively. Body mass index and Subscapular skinfold thickness were the two most important predictors of prediabetes/diabetes among adolescents. Further, physical activities show a negative association with pre-diabetes/ diabetes. Moreover, interaction models in the present study clearly reveal the fact that adolescent girls were less likely to suffer from pre-diabetes/diabetes than adolescent boys. Additionally, it was found that the prevalence of pre-diabetes/diabetes was high among adolescent girls from lower socio-economic strata.

Conclusion: The high prevalence of pre-diabetes and diabetes among adolescents portrayed serious public health concern in India. As body mass index and Subscapular skinfold thickness were positively associated with prediabetes/diabetes conditions among adolescents. Therefore, effective approaches are needed to be taken to tackle these pre-diabetes/diabetes conditions among adolescents and especially among adolescent boys.
\end{abstract}

Keywords: Pre-diabetes/diabetes, Obesity, Adolescents, India

\footnotetext{
* Correspondence: psmishrabhu@gmail.com

${ }^{2}$ Institute for Social and Economic Change, Bengaluru, Karnataka 560072,

India

Full list of author information is available at the end of the article
}

(c) The Author(s). 2021 Open Access This article is licensed under a Creative Commons Attribution 4.0 International License, which permits use, sharing, adaptation, distribution and reproduction in any medium or format, as long as you give appropriate credit to the original author(s) and the source, provide a link to the Creative Commons licence, and indicate if changes were made. The images or other third party material in this article are included in the article's Creative Commons licence, unless indicated otherwise in a credit line to the material. If material is not included in the article's Creative Commons licence and your intended use is not permitted by statutory regulation or exceeds the permitted use, you will need to obtain permission directly from the copyright holder. To view a copy of this licence, visit http://creativecommons.org/licenses/by/4.0/. The Creative Commons Public Domain Dedication waiver (http://creativecommons.org/publicdomain/zero/1.0/) applies to the data made available in this article, unless otherwise stated in a credit line to the data. 


\section{Background}

The International Diabetes Federation (IDF) estimated that 1.1 million children and adolescents aged 14-19 years were living with diabetes [1]. In addition, the increasing prevalence of pre-diabetes and diabetes led by obesity among the young population fuelled metabolic syndrome and obesity-related co-morbidities across the low and middle-income countries [2]. There is a global effort to curb down the rise of diabetes and obesity by 2025; however, a steady rise in the prevalence of diabetes is a matter of concern [3].

India alone contributes a larger proportion to premature deaths from non-communicable diseases (NCDs), including diabetes across all socio-economic groups [4]. Achieving the global agenda of Sustainable Development Goals (SDGs) by 2030, by reducing one-third of premature mortality from NCDs-including diabetes, a universal healthcare access approach is therefore required in most developing countries, including India [3, 4]. India is currently going through the nutritional transition, and it is evident with a rise in the prevalence of overweight and obesity [5]. Also, estimates shows that nearly 166 million adults are overweight/obese in 2016 in India [3]. In the case of adults who are affected by diabetes, the numbers are estimated to be nearly 73 million, the second-largest in the world [1].

Diabetes is a chronic, progressive disease characterized by elevated levels of blood glucose levels. It is also recognized as a complex disease that affects people of different ages due to different causes. There are three main types of diabetes, type-1 diabetes (T1D), type-2 diabetes (T2D), and gestational diabetes mellitus (GDM) [3]. The type-1 diabetes incidence rate is increasing among the younger population, and the contributing factors have remained unclear. However, there are factors that affect the heterogeneity of the respective disease such as genetic factors, behavioural risk factors, food and dietary habits, physical inactivity, lifestyle changes.

Furthermore, the prevalence of pre-diabetes/diabetes among children, adolescents, and even younger adults is increasing at a much higher rate irrespective of socio-economic strata, and it becomes more severe because of the rising levels of obesity among them [6-8], physical inactivity $[9,10]$, and poor dietary habits [11]. Moreover, increased levels of substance use $[12,13]$ among adolescent groups have also led to risk for prediabetes/diabetes-associated health issues [10, 14]. Further, the high spread of pre-diabetes/diabetes among the population is also witnessed in the light of different socio-economic groups [15], and it is concentrated differently across rural-urban, age-sex, and family history of diabetes [16-18].

However, lack of physical activities and having a sedentary lifestyle among adolescents made responsible for cardiovascular disease and obesity-related risk factors which in turn may cause menace of pre-diabetes/diabetes among them $[10,19,20]$. That has not been well established in the literature in the Indian context among the adolescents population. In this way, there are multiple determinants associated with risk factors for prediabetes and diabetes that influence the adolescent population and they are more prone to be affected by them [16, 21]. Moreover, Triglycerides, Cholesterol, High-density lipoprotein (HDL), and Low-density lipoprotein (LDL) levels are found to be significant factors for the prevalence of pre-diabetes/diabetes among adolescents [22, 23].

The present study utilized the different forms of obesity, which may affect the chaos of pre-diabetes/diabetes differently. Previous literature showed different measures of obesity in their studies [11, 24-26]. For instance, obesity measured through body mass index [23], waist circumference [23], waist to hip ratio [27], Subscapular skinfold thickness (SSFT) [28] and Triceps skinfold thickness (TSFT) [28].

There exists a dearth of literature focusing on the association of pre-diabetes/diabetes with obesity levels among adolescents at the national level in India. Therefore, the present study aims to estimate the prevalence of pre-diabetes/diabetes at the national level. Additionally, the study also analyses the factors that are responsible for pre-diabetes/diabetes conditions among adolescents (for both adolescent boys and girls) at the national level. The study hypothesized that there was no association between pre-diabetes/diabetes conditions with obesity levels and physical activity among adolescents in India.

\section{Methods}

The data was taken from the Comprehensive National Nutrition Survey (CNNS), the first-ever nationally representative nutrition survey of children and adolescents in India. The survey collected the data on the status of preschool (0-4 years), school-age children (5-9 years), and adolescents (10-19 years) through interviews, a comprehensive set of anthropometric measures, and biochemical indicators. The CNNS covered all 30 states of India using a multi-stage survey design for the selection of households and individuals aged 0-19 years. The data has been collected with the written consent of the participants. The survey was implemented under the guidance of the MoHFW, UNICEF, a Technical Advisory Group (TAG), and the Center for Disease Control (CDC). Data can be obtained on request from the Population Council (India office) website. The detailed methodology, sampling design, and data collection procedure were published in the survey report [29]. The CNNS collected data from a total of 38,060 children aged 0-4 
years, 38,355 children aged 5-9 years, and 35,830 adolescents age 10-19 years. The study used a sample size of 17,865 adolescent boys and 17,965 adolescent girls for the analysis.

\section{Outcome variable}

The outcome variable was pre-diabetes/diabetes conditions among adolescents aged 10-19years. The prediabetes/diabetes was diagnosed using glycosylated haemoglobin (HbA1c) [30]. The American Diabetes Association recommended glycated hemoglobin (HbA1c) as a possible substitute to fasting blood glucose for diagnosis of diabetes [31, 32]. Analysis of glycated hemoglobin $(\mathrm{HbA} 1 \mathrm{c})$ in blood provides the evidence about the respondent's/individual average blood glucose levels during the last 2-3 months, which is the predicted half-life of red blood cells (RBCs) [33]. The cut-off points for pre-diabetes/diabetes among adolescents were as: pre-diabetes $(>5.8 \%$ and $\leq 6.4 \%)$ and diabetes (> 6.4\%) [34]. We merged diabetes and pre-diabetes into one category due to the small sample in the diabetes condition. The outcome variable was divided into two categories as 0 "no pre-diabetes/diabetes" and 1 "prediabetes/diabetes". The type of diabetes studied is Type 2 Diabetes mellitus. The study did not include fasting glucose data as HbA1c reflects average plasma glucose levels over the previous 8 to 12 weeks [28].

\section{Exposure variables}

Body mass index was categorized as not overweight/ obese $(\mathrm{BMI} \leq+1 \mathrm{SD})$ and overweight/obese $(\mathrm{BMI}>+1$ $\mathrm{SD})$ group, abdominal obesity was categorized as no (waist circumference-for-age $\leq+1 \mathrm{SD}$ ) and yes (waist circumference-for-age $>+1 \mathrm{SD}$ ), Triceps skinfold thickness (TSFT) was grouped as not overweight (TSFT-forage $\leq+1$ SD) and overweight (TSFT-for-age $>+1$ SD) and Subscapular skinfold thickness (SSFT) was divided into two categories: not overweight (SSFT-for-age $\leq+1$ SD) and overweight (SSFT-for-age $>+1$ SD). Body mass index $z$-scores, also called BMI standard deviation (s.d.) scores, are measures of relative weight adjusted for child age and sex [35]. Given a child's age, sex, BMI, and an appropriate reference standard, a BMI $z$-score (or its equivalent BMI-for-age percentile) can be determined [35]. Physical activity was categorized as a low, medium, and high using the principal component analysis approach as 15 questions were used to generate the respective variable [29]. Serum triglycerides (assessed by spectrophotometry and enzymatic endpoint method) was categorized into low $(<130 \mathrm{mg} / \mathrm{dl})$ and high $(\geq 130$ $\mathrm{mg} / \mathrm{dl}$ ), cholesterol (assessed by spectrophotometry using cholesterol oxidase esterase peroxidase) was recoded as low $(<200 \mathrm{mg} / \mathrm{dl})$ and high $(\geq 200 \mathrm{mg} / \mathrm{dl})$, low-density lipoprotein (LDL) (assessed by spectrophotometry and direct measure cholesterol oxidase) was categorized as low $(<130 \mathrm{mg} / \mathrm{dl})$ and high $(\geq 130 \mathrm{mg} / \mathrm{dl})$, and highdensity lipoprotein (HDL) cholesterol (assessed by spectrophotometry and direct measure polyethylene glycolmodified cholesterol oxidase) was recoded as low $(<40$ $\mathrm{mg} / \mathrm{dl})$ and high $(\geq 40 \mathrm{mg} / \mathrm{dl})$. Age was categorized into early adolescents (10-14 years) and late adolescents (1519 years), education was divided into no schooling, dropouts and currently studying, Media exposure was categorized as no "no exposure to television \& radio \& newspaper \& internet" and yes "exposure to television or radio or newspaper or internet), parent education was recoded as (both uneducated, anyone educated and both educated), parent diabetes status was categorized as (no or anyone had), the diet was categorized as vegetarian and non-vegetarian; Junk food consumption was categorized as no, 1-2 days and 3-7 days; substance use was categorized as no and yes. Caste was categorized as Scheduled Caste/Scheduled Tribe (SC/ST) and nonScheduled Caste/Scheduled Tribe. Among the four categories of castes, people belonging to the Scheduled caste lies at the bottom of the Indian caste system and have been exploited for over centuries. The indigenous groups of India belong to the category of Scheduled tribes and are among the most deprived sections. Therefore SC/ST is the deprived section of the society [36]. Religion was categorized as Hindu, Muslim, Christian, and Others; wealth quintile was categorized as poor, middle and rich; the residence was categorized as urban and rural; and region were divided as north, central, east, north-east, west, and south.

\section{Statistical analysis}

Descriptive analysis was used to show the sample profile of the respondents. Further, bivariate analysis was carried out to estimate the prevalence of pre-diabetes/diabetes by selected variables, and a proportion test was applied to test the significant difference between adolescent boys and girls for pre-diabetes/diabetes. Finally, multivariate logistic regression was performed to find out the effects of different predictors on pre-diabetes/ diabetes. The results are presented in the form of an odds ratio (OR) with a $95 \%$ confidence interval $(\mathrm{CI})$. The model is usually put into a more compact form as follows:

$$
\ln \left(\frac{P_{i}}{1-P_{i}}\right)=\beta_{0}+\beta_{1} x_{1}+\ldots+\beta_{M} x_{M},
$$

Where $\beta_{0}, \ldots ., \beta_{M}$ are the regression coefficient indicating the relative effect of a particular explanatory variable on the outcome, the study further examined the possible interaction between body mass index, abdominal obesity, triceps skinfold thickness (TSFT), 
subscapular skinfold thickness (SSFT), and gender in models $2,3,4$, and 5 respectively.

\section{Results}

Anthropometric and socio-demographic profiles of adolescents aged 10-19 years are presented in Table 1 . Overall, nearly $5 \%$ of adolescent boys and girls (each) were overweight/obese whereas about $2 \%$ and $1 \%$ of adolescent boys and girls had abdominal obesity, respectively. Moreover, about $5 \%$ and $2 \%$ of adolescent boys and girls had triceps skinfold thickness, respectively. Seven percent of adolescent boys and $4 \%$ of girls had subscapular skinfold thickness. About $14 \%$ of boys and $18 \%$ of adolescent girls had high serum triglycerides. Moreover, almost the same proportion of adolescents (3\% vs. $5 \%$ ) had high cholesterol and low-density lipoprotein respectively. The proportion of adolescents was higher in the 10-14 years age group irrespective of gender. The majority of adolescents are currently studying and, around $17 \%$ of adolescent boys and $21 \%$ of girls were school dropouts. More than three-fourth of adolescents had some mass media exposure. One-fourth of the parents had no schooling and a very low proportion of parents (anyone) had diabetes (4\%). About $81 \%$ of adolescent boys and $73 \%$ of girls were non-vegetarian. Seven out of ten adolescents were consumed 1-2 days of junk food. Substance use was higher among adolescent boys (8\%) than girls. One-third of adolescents belonged to SC/ST group and the majority of adolescents were Hindu. Two-fifth of adolescents belonged to rich families and one-fourth of adolescents lived in urban areas.

Gender differentials for pre-diabetes/diabetes among adolescents are presented in Fig. 1. Pre-diabetes/diabetes was more prevalent among 15 years of adolescents, and it is lower in 10 years of adolescents. Though, the prevalence of pre-diabetes/diabetes was higher among adolescent boys compared to girls irrespective of their age.

Table 2 shows the gender differences in the prevalence of pre-diabetes/diabetes among adolescents aged 10-19 years across different socio-demographic characteristics in India. The prevalence of pre-diabetes/diabetes was higher among adolescent boys across all the selected socio-demographic characteristics and differences were statistically significant. Substantial differences were observed among adolescents who had abdominal obesity (11.7\%), overweight (SSFT) (9.8\%), belonged to the Northeast region (10.1\%), consumed junk food for 3-7 days $(8.5 \%)$, and belonged to the middle $(6.4 \%)$ and rich families (5.6\%).

Binary logistic regression estimates for pre-diabetes/ diabetes among adolescents aged 10-19 years are presented in Table 3. Results from model-1 found that overweight/obese adolescents had $41 \%$ higher chances [Odd ratio (OR), 1.41; CI: $1.08-1.82$ ] to get pre- diabetes/diabetes compared to adolescents who were not overweight/obese. Moreover, SSFT had a significant effect on pre-diabetes/diabetes among adolescents. Overweight adolescents (SSFT) were significantly more likely (OR, 1.49: CI: 1.15-1.94) to suffer from pre-diabetes/diabetes than those who were not overweight. There was a negative relationship between the physical activity of adolescents and their pre-diabetes/diabetes condition. Adolescents involved in high physical activity were less likely to suffer from pre-diabetes/diabetes compared to those who did low physical activities. However, the result was not significant for physical activities.

Model 2 represents the interaction between body mass index and gender for pre-diabetes/diabetes. Interestingly, adolescent girls with not overweight/obese category were significantly less likely to suffer (OR, 0.72; CI: 0.62-0.83) from pre-diabetes/diabetes compared to adolescent boys who belonged to the not overweight/obese category. Interaction between gender and abdominal obesity for pre-diabetes/diabetes condition is presented in Model 3. Adolescent boys having abdominal obesity were more likely (OR, 1.26; CI: $0.74-2.17$ ) to suffer from prediabetes/diabetes than adolescent boys with no abdominal obesity. Moreover, adolescent girls with abdominal obesity were significantly less likely (OR, 0.73 ; CI: $0.63-$ $0.84)$ to suffer from pre-diabetes/diabetes compared to adolescent boys with no abdominal obesity. Results from Model 4 revealed the interaction between gender and triceps skinfold thickness (TSFT) for pre-diabetes/diabetes among adolescents. The likelihood of pre-diabetes/diabetes among overweight (TSFT) adolescent boys was lower (OR, 0.96; CI: 0.63-1.45) than a not overweight adolescent boys. However, the chances of pre-diabetes/ diabetes among girls with not overweight category were significantly lower (OR, 0.72 ; CI: $0.62-0.83$ ) compared to boys with no overweight category. Model 5 shows the interaction between gender and subscapular skinfold thickness (SSFT) for pre-diabetes/diabetes among adolescents. SSFT had a significant effect on diabetes among both adolescent boys and girls. The probability of getting diabetes was significantly higher (OR, 2.23; CI: 1.533.23) among overweight (SSFT) adolescent boys than those who were not overweight. Moreover, adolescent girls from the not overweight category were significantly less likely (OR, 0.72; CI: $0.62-0.84$ ) to suffer from prediabetes/diabetes compared to boys from the not overweight category.

\section{Discussion}

The overall prevalence of pre-diabetes/diabetes was $12.3 \%$ and $8.4 \%$ among adolescent boys and girls in India respectively. Moreover, it was found that body mass index (BMI) and subscapular skinfold thickness (SSFT) was significantly associated with pre-diabetes/diabetes 
Table 1 Anthropometric and socio-demographic profile of adolescents aged 10-19years in India, 2016-18

\begin{tabular}{l} 
Variables \\
\hline Body mass Index (BMI) \\
Not overweight/obese \\
Overweight/obese \\
Abdominal obesity
\end{tabular}

$$
\text { No }
$$$$
\text { Yes }
$$

Triceps skinfold thickness (TSFT)

$$
\text { Not overweight }
$$$$
\text { Overweight }
$$

Subscapular skinfold thickness (SSFT)

$$
\begin{aligned}
& \text { Not overweight } \\
& \text { Overweight }
\end{aligned}
$$

\section{Physical activity}

Low

Medium

High

\section{Serum triglycerides}

$\begin{array}{ll}\text { Low } & 5856 \\ \text { High } & 963\end{array}$

\section{Cholesterol}

Low

High

Low-density lipoprotein

$$
\text { Low }
$$

High

High-density lipoprotein

$$
\text { Low }
$$

\section{Age (years)}

Early adolescence (10-14)

Late adolescence (15-19)

\section{Education}

$$
\begin{aligned}
& \text { No schooling } \\
& \text { Dropout } \\
& \text { Currently studying }
\end{aligned}
$$

234

Media exposure

No

Yes

Both uneducated

Anyone educated

Both educated

\section{Adolescent boys}

15,697

7,116

16,675

337

6,356

1249

6963

4975

6486

(1)

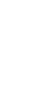

.

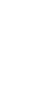

Parents diabetes status

8030

\section{Adolescent girls}

Sample

Percent

14,841

95.1

761

4.9

16,271

98.8

202

1.2

16,276

98.3

276

15,826

95.7

704

12,178

70.0

2544

14.6

27.0

2683

15.4

5241

81.7

1172

18.3

14.1

96.6

6112

95.4

3.4

293

4.6

6121

95.5

96.8

289

1533

24.0

29.6

4854

8573

1182

3721

21.4

17.3

12,502

71.8

77.9

4080

23.4

16.0

13,325

76.6

4529

26.0

5782

33.2

7094 
Table 1 Anthropometric and socio-demographic profile of adolescents aged 10-19years in India, 2016-18 (Continued)

\begin{tabular}{|c|c|c|c|c|}
\hline \multirow[t]{2}{*}{ Variables } & \multicolumn{2}{|c|}{ Adolescent boys } & \multicolumn{2}{|c|}{ Adolescent girls } \\
\hline & Sample & Percent & Sample & Percent \\
\hline No one & 17,675 & 95.9 & 16,769 & 96.4 \\
\hline Anyone had & 750 & 4.1 & 636 & 3.7 \\
\hline \multicolumn{5}{|l|}{ Diet } \\
\hline Vegetarian & 3519 & 19.1 & 4770 & 27.4 \\
\hline Non-vegetarian & 14,902 & 80.9 & 12,634 & 72.6 \\
\hline \multicolumn{5}{|c|}{ Junk food consumption } \\
\hline No & 4876 & 26.5 & 4638 & 26.7 \\
\hline $1-2$ days & 12,771 & 69.3 & 12,216 & 70.2 \\
\hline $3-7$ days & 774 & 4.2 & 549 & 3.2 \\
\hline \multicolumn{5}{|l|}{ Substance use } \\
\hline No & 16,876 & 91.6 & 17,194 & 98.8 \\
\hline Yes & 1549 & 8.4 & 211 & 1.2 \\
\hline \multicolumn{5}{|l|}{ Caste } \\
\hline SC/ST & 5769 & 33.2 & 5769 & 33.2 \\
\hline Non-SC/ST & 11,636 & 66.9 & 11,636 & 66.9 \\
\hline \multicolumn{5}{|l|}{ Religion } \\
\hline Hindu & 14,878 & 80.8 & 13,797 & 79.3 \\
\hline Muslim & 2643 & 14.3 & 2819 & 16.2 \\
\hline Christian & 458 & 2.5 & 381 & 2.2 \\
\hline Others & 446 & 2.4 & 408 & 2.3 \\
\hline \multicolumn{5}{|l|}{ Wealth quintile } \\
\hline Poor & 7292 & 39.6 & 7036 & 40.4 \\
\hline Middle & 3696 & 20.1 & 3473 & 20.0 \\
\hline Rich & 7437 & 40.4 & 6896 & 39.6 \\
\hline \multicolumn{5}{|l|}{ Place of residence } \\
\hline Rural & 13,788 & 74.8 & 13,174 & 75.7 \\
\hline Urban & 4637 & 25.2 & 4231 & 24.3 \\
\hline \multicolumn{5}{|l|}{ Regions } \\
\hline North & 2597 & 14.1 & 2306 & 13.3 \\
\hline Central & 5750 & 31.2 & 5936 & 34.1 \\
\hline East & 4248 & 23.1 & 4148 & 23.8 \\
\hline Northeast & 582 & 3.2 & 507 & 2.9 \\
\hline West & 2327 & 12.6 & 1864 & 10.7 \\
\hline South & 2921 & 15.9 & 2644 & 15.2 \\
\hline Total & 17,865 & 100.0 & 17,965 & 100.0 \\
\hline
\end{tabular}

SC/ST Scheduled caste/ Scheduled tribe

among adolescents in India. Additionally, it was revealed that adolescent girls were less likely to suffer from prediabetes/diabetes than adolescent boys in every category of obesity measure.

Earlier studies found that no accessibility of proper care such as early diagnosis, diabetes care management, and blood glucose testing available in primary healthcare settings in the early phases of life for children and adolescents $[16,37]$. Early diagnosis of pre-diabetes/diabetes can have lesser risk in the long-term than remaining undiagnosed.

The current study reveals the fact that there was a positive association between BMI, SSFT, and low physical activity status with pre-diabetes/diabetes conditions 


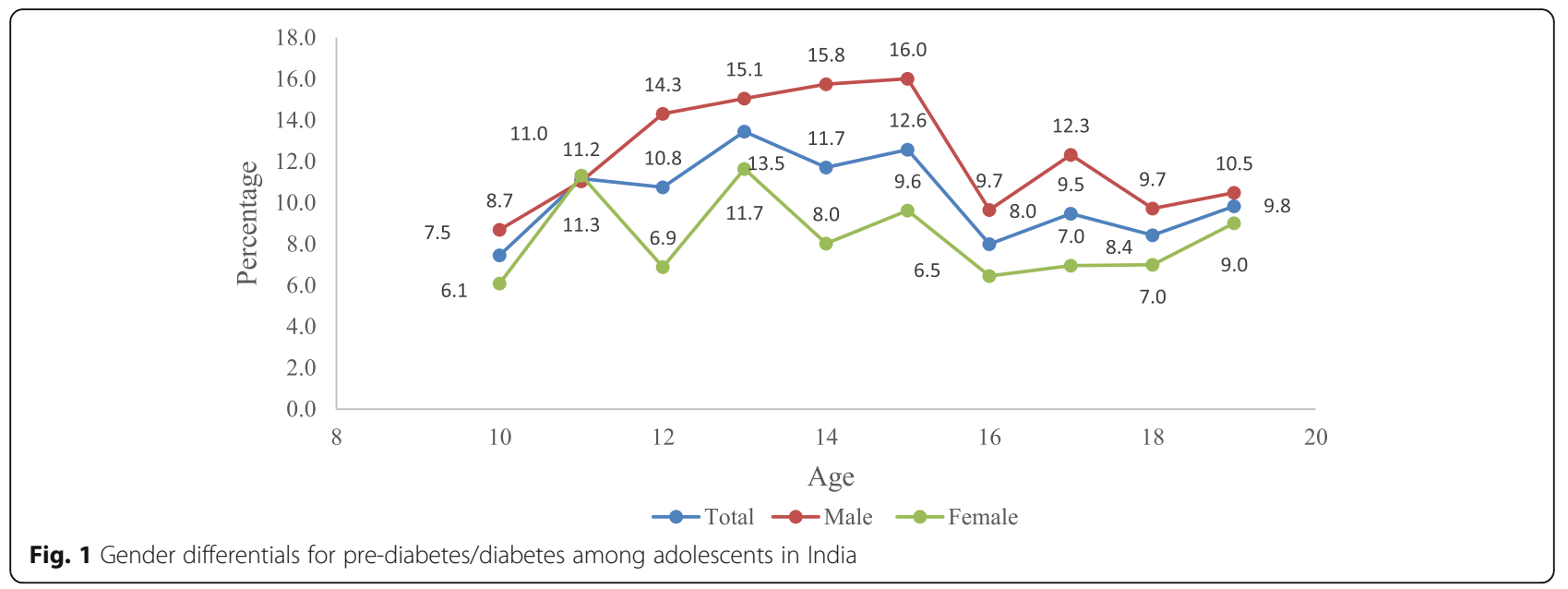

among adolescents in India. The results were consistent with previous findings that increased BMI was positively associated with pre-diabetes/diabetes conditions among adolescents [20]. Moreover, SSFT was also a significant risk factor of pre-diabetes/diabetes conditions among adolescents, as argued by previous literature [38-40]. Apart from obesity, genetic and metabolic factors, there are other significant risk factors, but in the present study, those were not taken into account due to data limitations [2, 26, 37]. Additionally, low physical activity status was found to be a substantial risk factor for prediabetes/diabetes conditions among adolescents, as found in previous studies [41]. It was too argued that even a moderate level of physical activity could help in the prevention of pre-diabetes conditions by reducing obesity levels among adolescents [41].

Dietary practices with unhealthy food such as high intake of junk foods and sugar-sweetened beverages are associated with a high prevalence of obesity/overweight that may lead to pre-diabetes/diabetes conditions among adolescents $[42,43]$. This finding is consistent with findings of the present that adolescents with junk food consumption frequency of 3-7 days a week had a high prevalence of pre-diabetes/diabetes condition. Additionally, the menace of substance use among adolescents was a staunch factor for pre-diabetes/diabetes conditions. The finding was paralleled with the previous study that abnormal alcohol and drug use among adolescents increases the risk of pre-diabetes/diabetes conditions [44].

Although it was earlier argued that diabetes is an affluent disease and it occurs among the population from higher socio-economic strata [16], however, the present study revealed that the disease pattern is now shifting towards lower affluent population, for instance, prediabetes/diabetes condition was more among SC/ST, poor and adolescents from rural areas especially in case of adolescent girls. Further research is needed to look into the highly prevailing pre-diabetes/diabetes among adolescents irrespective of caste, class, gender and geographical locations.

The results showed that pre-diabetes/diabetes vary across gender; for instance, gender differential was over $4 \%$, i.e., adolescent boys are more prone to pre-diabetes/ diabetes than adolescent girls. Moreover, for most of the background factors, adolescent boys were at high risk than adolescent girls. Even previous literature argued that adolescent girls do have a lower probability to suffer from pre-diabetes/diabetes [45]. The plausible reasons can be derived through descriptive statistics in the present study where it was clearly visible that obesity levels and substance use were low among adolescent girls and which may further protect them from the burden of pre-diabetes and diabetes condition in comparison to their counterpart. Additionally, interaction models revealed that adolescent girls were less likely to suffer from pre-diabetes/diabetes in comparison to adolescent boys for the obesity measure. The findings are found to be consistent with the previous one as it was argued that adolescent girls are less likely to be overweight or obese [46].

\section{Limitation}

The study has potential limitations. This study used crosssectional data that does not allow establishing a causal relationship between variables. Additionally, Type 1 Diabetes mellitus, which is more common in adolescents, is not taken into account which is also a major limitation of this article. Glucose Tolerance Test, Fasting Blood Sugar (FBS) test and Post Prandial Blood Sugar (PPBS) test were not performed. Despite the limitation, the study also has its own strength. This study used large scale survey data of adolescents which is the first-ever survey on nutrition and gives reliable estimates on diabetes at the national level. 
Table 2 Prevalence of pre-diabetes/diabetes among adolescents aged 10-19years by socio-demographic characteristics in India, 2016-18

\begin{tabular}{lllll}
\hline Variables & Adolescent boys & Adolescent girls & Adolescent boys - Adolescent girls & $\begin{array}{l}\text { Proportion Test } \\
\end{array}$ \\
& $(\%)$ & $(\%)$ & $(\%)$ & $P<0.05$ \\
\hline
\end{tabular}

Body Mass Index (BMI)

$\begin{array}{llll}\text { Not overweight/obese } & 12.1 & 8.2 & 3.9 \\ \text { Overweight/obese } & 17.2 & 11.5 & 5.7\end{array}$

Abdominal obesity

12.2
No
Yes

Triceps skinfold thickness (TSFT)

$\begin{array}{lr}\text { Not overweight } & 12.0 \\ \text { Overweight } & 18.6\end{array}$

$\begin{array}{ll}8.3 & 3.8 \\ 9.1 & 11.7\end{array}$

Subscapular skinfold thickness (SSFT)

$\begin{array}{ll}\text { Not overweight } & 11.6 \\ \text { Overweight } & 22.6\end{array}$

$1.6 \quad 8.1$

Overweight

Physical activity

$\begin{array}{ll}\text { Low } & 13.6 \\ \text { Medium } & 12.2 \\ \text { High } & 11.6\end{array}$

$\begin{array}{ll}8.3 & 5.4 \\ 9.2 & 3.0 \\ 7.5 & 4.0\end{array}$

Serum triglycerides

Low

High

\section{Cholesterol}

Low

High

Low-density lipoprotein

Low

High

High-density lipoprotein

$\begin{array}{ll}\text { Low } & 8.7 \\ \text { High } & 12.3\end{array}$

Age (years)

Early adolescence (10-14) 13.0

Late adolescence (15-19) 11.7

Education

No schooling

8.2

Dropout

Currently studying

12.6

Media exposure

No

Yes

12.6

Parent education

Both uneducated

Anyone educated

Both educated

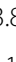

1.7

8.0

3.5

9.8

4.0

0


Table 2 Prevalence of pre-diabetes/diabetes among adolescents aged 10-19years by socio-demographic characteristics in India, 2016-18 (Continued)

\begin{tabular}{|c|c|c|c|c|}
\hline Variables & $\begin{array}{l}\text { Adolescent boys } \\
(\%)\end{array}$ & $\begin{array}{l}\text { Adolescent girls } \\
\text { (\%) }\end{array}$ & $\begin{array}{l}\text { Adolescent boys - Adolescent girls } \\
(\%)\end{array}$ & $\begin{array}{l}\text { Proportion Test } \\
P<0.05\end{array}$ \\
\hline \multicolumn{5}{|c|}{ Parents diabetes status } \\
\hline No one & 12.5 & 8.4 & 4.2 & * \\
\hline Anyone had & 10.2 & 6.7 & 3.5 & \\
\hline Diet & & & 0.0 & \\
\hline Vegetarian & 14.1 & 10.3 & 3.9 & * \\
\hline Non-vegetarian & 12.0 & 7.6 & 4.5 & * \\
\hline \multicolumn{5}{|c|}{ Junk food consumption } \\
\hline No & 12.9 & 10.7 & 2.2 & * \\
\hline $1-2$ days & 12.1 & 7.5 & 4.7 & * \\
\hline 3-7 days & 14.1 & 5.7 & 8.5 & * \\
\hline \multicolumn{5}{|l|}{ Substance use } \\
\hline No & 11.9 & 8.2 & 3.7 & * \\
\hline Yes & 18.9 & 14.1 & 4.8 & * \\
\hline \multicolumn{5}{|l|}{ Caste } \\
\hline SC/ST & 11.8 & 9.5 & 2.4 & * \\
\hline Non-SC/ST & 12.7 & 7.7 & 5.0 & * \\
\hline \multicolumn{5}{|l|}{ Religion } \\
\hline Hindu & 12.3 & 8.5 & 3.8 & * \\
\hline Muslim & 11.8 & 6.3 & 5.5 & * \\
\hline Christian & 18.1 & 12.3 & 5.8 & * \\
\hline Others & 15.3 & 10.1 & 5.1 & \\
\hline \multicolumn{5}{|l|}{ Wealth quintile } \\
\hline Poor & 10.8 & 9.4 & 1.4 & * \\
\hline Middle & 14.0 & 7.6 & 6.4 & * \\
\hline Rich & 13.3 & 7.6 & 5.6 & * \\
\hline \multicolumn{5}{|l|}{ Place of residence } \\
\hline Rural & 12.4 & 8.4 & 4.0 & * \\
\hline Urban & 12.7 & 8.0 & 4.7 & * \\
\hline \multicolumn{5}{|l|}{ Regions } \\
\hline North & 16.3 & 10.6 & 5.8 & * \\
\hline Central & 11.1 & 6.9 & 4.2 & * \\
\hline East & 9.3 & 5.5 & 3.7 & * \\
\hline Northeast & 18.4 & 8.3 & 10.1 & * \\
\hline West & 13.7 & 12.9 & 0.9 & \\
\hline South & 14.5 & 10.2 & 4.3 & * \\
\hline Total & 12.4 & 8.3 & 4.1 & * \\
\hline
\end{tabular}

*if $p<0.05 ;$ SC/ST Scheduled caste/ Scheduled tribe, \% Percentage

\section{Conclusion}

Our study found that pre-diabetes/diabetes was highly prevalent among adolescents especially among adolescent boys in India. Interaction models in the present study clearly reveal the fact that adolescent girls are less likely to suffer from pre-diabetes/diabetes than adolescent boys. BMI and SSFT are the two most important predictors of pre-diabetes/diabetes among adolescents in India. The high prevalence of pre-diabetes/diabetes among adolescent girls from lower socio-economic 
Table 3 Logistic regression estimates for pre-diabetes/diabetes among adolescents aged 10-19 by socio-demographic characteristics in India, 2016-18

\begin{tabular}{|c|c|c|c|c|c|}
\hline Variables & $\begin{array}{l}\text { Model-1 } \\
\text { OR(95\% Cl) }\end{array}$ & $\begin{array}{l}\text { Model-2 } \\
\text { OR(95\% Cl) }\end{array}$ & $\begin{array}{l}\text { Model-3 } \\
\text { OR(95\% Cl) }\end{array}$ & $\begin{array}{l}\text { Model-4 } \\
\text { OR(95\% Cl) }\end{array}$ & $\begin{array}{l}\text { Model-5 } \\
\text { OR(95\% Cl) }\end{array}$ \\
\hline \multicolumn{6}{|l|}{ Body mass index (BMI) } \\
\hline Not overweight/obese & Ref. & & Ref. & Ref. & Ref. \\
\hline Overweight/obese & $1.41 *(1.08,1.82)$ & & $1.4^{*}(1.08,1.82)$ & $1.39 *(1.07,1.81)$ & $1.39 *(1.07,1.81)$ \\
\hline \multicolumn{6}{|l|}{ Abdominal obesity } \\
\hline No & Ref. & Ref. & & Ref. & Ref. \\
\hline Yes & $1.06(0.73,1.54)$ & $1.09(0.75,1.59)$ & & $1.07(0.73,1.55)$ & $1.07(0.74,1.56)$ \\
\hline \multicolumn{6}{|c|}{ Triceps skinfold thickness (TSFT) } \\
\hline Not overweight & Ref. & Ref. & Ref. & & Ref. \\
\hline Overweight & $0.89(0.64,1.24)$ & $0.92(0.66,1.27)$ & $0.9(0.65,1.25)$ & & $0.92(0.66,1.28)$ \\
\hline \multicolumn{6}{|c|}{ Subscapular skinfold thickness (SSFT) } \\
\hline Not overweight & Ref. & Ref. & Ref. & Ref. & \\
\hline Overweight & $1.49^{*}(1.15,1.94)$ & $1.49^{*}(1.15,1.94)$ & $1.5^{*}(1.15,1.95)$ & $1.5^{*}(1.15,1.95)$ & \\
\hline \multicolumn{6}{|l|}{ Physical activity } \\
\hline Low & Ref. & Ref. & Ref. & Ref. & Ref. \\
\hline Medium & $0.90(0.76,1.06)$ & $0.90(0.76,1.06)$ & $0.90(0.76,1.06)$ & $0.90(0.76,1.06)$ & $0.90(0.76,1.06)$ \\
\hline High & $0.80(0.85,1.14)$ & $0.80(0.85,1.14)$ & $0.80(0.85,1.14)$ & $0.80(0.84,1.14)$ & $0.80(0.85,1.14)$ \\
\hline \multicolumn{6}{|l|}{ Serum triglycerides } \\
\hline Low & Ref. & Ref. & Ref. & Ref. & Ref. \\
\hline High & $0.90(0.75,1.07)$ & $0.90(0.75,1.07)$ & $0.90(0.75,1.07)$ & $0.90(0.76,1.07)$ & $0.90(0.75,1.07)$ \\
\hline \multicolumn{6}{|l|}{ Cholesterol } \\
\hline Low & Ref. & Ref. & Ref. & Ref. & Ref. \\
\hline High & $1.02(0.69,1.52)$ & $1.03(0.69,1.53)$ & $1.03(0.69,1.52)$ & $1.03(0.69,1.53)$ & $1.03(0.69,1.53)$ \\
\hline \multicolumn{6}{|l|}{ Low-density lipoprotein } \\
\hline Low & Ref. & Ref. & Ref. & Ref. & Ref. \\
\hline High & $1.33(0.93,1.9)$ & $1.32(0.92,1.9)$ & $1.33(0.93,1.9)$ & $1.33(0.93,1.91)$ & $1.33(0.92,1.9)$ \\
\hline \multicolumn{6}{|l|}{ High-density lipoprotein } \\
\hline Low & Ref. & Ref. & Ref. & Ref. & Ref. \\
\hline High & $0.93(0.8,1.07)$ & $0.93(0.8,1.07)$ & $0.93(0.8,1.07)$ & $0.93(0.8,1.07)$ & $0.93(0.8,1.07)$ \\
\hline
\end{tabular}

Body mass index (BMI)*Gender

Adolescent boys ${ }^{\mathrm{a}}$ Not overweight/obese

Adolescent boys ${ }^{\mathrm{a}}$ Overweight/obese

Adolescent girls ${ }^{\text {a }}$ Not overweight/obese

Adolescent girls a Overweight/obese

Abdominal obesity*Gender

Adolescent boys ${ }^{a} \mathrm{No}$

Adolescent boys ${ }^{\mathrm{a}}$ Yes

Adolescent girls ${ }^{\mathrm{a}}$ No

Adolescent girls ${ }^{a}$ Yes

Triceps skinfold thickness (TSFT)*Gender

Adolescent boys ${ }^{\mathrm{a}}$ Not overweight

Adolescent boys ${ }^{\mathrm{a}}$ Overweight

Adolescent girls ${ }^{\text {a }}$ Not overweight
Ref.

$1.18(0.78,1.79)$

$0.72 *(0.62,0.83)$

$0.74(0.48,1.15)$
Ref.

$1.26(0.74,2.17)$

$0.73^{*}(0.63,0.84)$

$0.41(0.16,1.07)$
Ref.

$0.96(0.63,1.45)$

$0.72 *(0.62,0.83)$ 
Table 3 Logistic regression estimates for pre-diabetes/diabetes among adolescents aged 10-19 by socio-demographic characteristics in India, 2016-18 (Continued)

\begin{tabular}{|c|c|c|c|c|c|}
\hline \multirow[t]{2}{*}{ Variables } & Model-1 & Model-2 & Model-3 & Model-4 & Model-5 \\
\hline & OR(95\% Cl) & $\mathrm{OR}(95 \% \mathrm{Cl})$ & $\mathrm{OR}(95 \% \mathrm{Cl})$ & OR(95\% Cl) & OR(95\% Cl) \\
\hline Adolescent girls ${ }^{a}$ Overweight & & & & $0.48(0.21,1.1)$ & \\
\hline \multicolumn{6}{|c|}{ Subscapular skinfold thickness (SSFT)*Gender } \\
\hline Adolescent boys ${ }^{\mathrm{a}}$ Not overweight & & & & & Ref. \\
\hline Adolescent boys ${ }^{\mathrm{a}}$ Overweight & & & & & $2.23^{*}(1.53,3.23)$ \\
\hline Adolescent girls ${ }^{a}$ Not overweight & & & & & $0.72 *(0.62,0.84$ \\
\hline Adolescent girls ${ }^{a}$ Overweight & & & & & $1.38(0.9,2.11)$ \\
\hline
\end{tabular}

${ }^{a}$ Interaction; OR: Odds Ratio; Cl: Confidence Interval; Ref: Reference; ${ }^{*}$ if $p<0.05$

Note: All the models have been run after controlling the other socio-demographic factors

strata poses a high concern in the Indian scenario. The emerging type 1 diabetes (T1D) among children and adolescents cannot be prevented with existing knowledge [3]. However, type 2 diabetes can be prevented with effective approaches available, and premature deaths can be curtailed off [3]. To avoid diabetes and related risk factors, regular exercise, maintaining a healthy diet, avoiding substance use, and controlling blood pressure and lipids as recommended by WHO guidelines [3]. The present study also suggests that to prevent pre-diabetes/ diabetes conditions among adolescents certain health interventions are required. Utilization of knowledge, awareness, and programme (KAP) re-intervention to control obese/overweight among adolescents on the one hand and the regular health check-ups among adolescents, on the other hand, will surely help to curtail prediabetes/diabetes conditions among adolescents in India. Adolescent sub-populations are out of the coverage of the special programme that addresses the crucial public health issues of diabetes and related various risk factors.

\section{Abbreviations}

MoHFW: Ministry of Health and Family Welfare; UNICEF: United Nations Children Funds; CNNS: Comprehensive National Nutrition Survey; BMI: Body Mass Index; HbA1c: Glycosylated haemoglobin; T1D: Type-1 diabetes; T2D: Type-2 diabetes; GDM: Gestational diabetes mellitus; TSFT: Triceps skinfold thickness; SSFT: Subscapular skinfold thickness; LDL: Low-density lipoprotein; HDL: High-density lipoprotein; SC/ST: Scheduled Tribe/Scheduled Caste

\section{Acknowledgments}

The authors are thankful to the Population Council (India) for providing the data for this study. The authors are also thankful to Aditya and Megha Mittal for providing financial support to carry out the CNNS. Their generous support enabled us to utilize the highest quality and innovative methods for conducting the world's largest comprehensive nutrition survey. The authors also thankful to Dr. Sahana Nagaraj for her insightful comments and suggestions to improve the quality of the paper.

\section{Authors' contributions}

The concept was drafted by SS; PK and SS contributed to the analysis design; PSM and SS advised on the paper and assisted in paper conceptualization; PSM, PK, and SS contributed to the comprehensive writing of the article. All authors read and approved the final manuscript.
Funding

Authors did not receive any funding to carry out this research.

\section{Availability of data and materials}

We have provided details of the data in the methodology section. The CNNS data can be obtained on request from the Population Council, Delhi (India). The report and the survey tools are also available on the website: https:// www.popcouncil.org/uploads/pdfs/2019RH_CNNSreport.pdf and cnns. pc@gmail.com respectively.

\section{Declarations}

Ethics approval and consent to participate

The study is based on secondary data which is publically available on request from cnns.pc@gmail.com. Therefore ethical approval and consent to participate are not required.

\section{Consent for publication}

Not applicable.

\section{Competing interests}

The authors declare that they have no competing interests.

\section{Author details}

${ }^{1}$ International Institute for Population Sciences, Mumbai, Maharashtra 400088 India. ${ }^{2}$ Institute for Social and Economic Change, Bengaluru, Karnataka 560072, India. ${ }^{3}$ Vaidyaratmam Group, Thrissur, India.

Received: 24 July 2020 Accepted: 21 June 2021

Published online: 07 July 2021

\section{References}

1. IDF. IDF DIABETES ATLAS Eighth edition 2017; 2017. Epub ahead of print 2017. https://doi.org/10.1016/S0140-6736(16)31679-8.

2. Tester J, Sharma S, Bradner C, et al. Diabetes \& Metabolic Syndrome : Clinical Research \& Reviews Gender differences in prediabetes and insulin resistance among 1356 obese children in northern California. Diabetes Metab Syndr Clin Res Rev. 2013;7(3):161-5. https://doi.org/10.1016/j.dsx.2 013.06.002.

3. WHO. Consideration of the evidence on childhood obesity for the Commission on Ending Childhood Obesity Report of the Ad hoc Working Group on Science and Evidence for Ending Childhood Obesity. Geneva: World Health Organization; 2016. p. 1-218.

4. Dandona L, India State-Level Disease Burden Initiative Collaborators (167 authors). Nations within a nation : variations in epidemiological transition across the states of India , 1990-2016 in the Global Burden of Disease Study. Lancet. 2017;390(10111):2437-60. https://doi.org/10.1016/S0140-673 6(17)32804-0

5. IIPS and ICF. National Family Health Survey (NFHS-4), 2015-16. Mumbai, India. 2017.

6. Chatterjee S, Khunti K, Davies MJ. Type 2 diabetes. Lancet. 2017;6736:1-13.

7. Ramachandran A, Snehalatha C, Kapur A, Vijay V, Mohan V, Das AK, et al. High prevalence of diabetes and impaired glucose tolerance in India : 
National Urban Diabetes Survey. Diabetologia. 2001;44(9):1094-101. https:// doi.org/10.1007/s001250100627.

8. Hills PAP, Arena PR, Kamlesh P, et al. Series type 2 diabetes in South Asia 1 epidemiology and determinants of type 2 diabetes in. Lancet Diabetes Endocrinol. 2018;8587:1-13.

9. Hills AP, Andersen LB, Byrne NM. Physical activity and obesity in children; 2011. p. 866-70

10. Kumar B, Robinson R, Till S. Physical activity and health in adolescence. Clin Med. 2015;15:267-72.

11. Lahiri A, Chakraborty A, Dasgupta U, Roy AKS, Bhattacharyya K. Effect of dietary habit and physical activity on Overnutrition of Schoolgoing adolescents : a longitudinal assessment in a rural block of West Bengal. Indian J Public Health. 2019;63(3):171-7. https://doi.org/10.4103/ijph.IJPH_1 59_19.

12. Ramachandran A, Snehalatha C, Mary S, Mukesh B, Bhaskar AD, Vijay V. The Indian Diabetes Prevention Programme shows that lifestyle modification and metformin prevent type 2 diabetes in Asian Indian subjects with impaired glucose tolerance ( IDPP-1 ). Diabetologia. 2006;49:289-97. https:// doi.org/10.1007/s00125-005-0097-z

13. Ujuanbi SA, Mezie-Okoye MM. Prevalence of overaweight and obeisty among adolescents in secondary schools in an urban city in Niger Delta region, Nigeria. Nig Del Med J. 2019;3(4):22-30.

14. Ojo O, Wang X, Ojo OO, et al. The effects of substance abuse on blood glucose parameters in patients with diabetes : a systematic review and Meta-analysis. Int J Environ Res Public Heal. 2018;15:1-16.

15. Vennu V, Abdulrahman TA, Bindawas SM. The prevalence of overweight, obesity, hypertension, and diabetes in India: analysis of the 2015-2016 national family health survey. Int J Environ Res Public Heal. 2019;16:1-22.

16. Anjana RM, Deepa M, Pradeepa $R$, et al. Prevalence of diabetes and prediabetes in 15 states of India : results from the ICMR - INDIAB population-based cross-sectional study. Lancet Diabetes Endocrinol. 2017;5: 585-96.

17. Gupta N, Shah P, Nayyar S, Misra A. Childhood obesity and the metabolic syndrome in developing countries. Indian J Pediatr. 2013;80(S1):28-37. https://doi.org/10.1007/s1 2098-012-0923-5.

18. Patterson CC, Dahlquist GG, Gyürüs E, et al. Incidence trends for childhood type 1 diabetes in Europe during 1989-2003 and predicted new cases 2005-20 : a multicentre prospective registration study. Lancet. 2005;373: 2027-33.

19. Srinivasan M. Sudha, Pescatello S. Linda BNA. Current perspectives on physical activity and exercise recommendations for children Spectrum disorders. Am Phys Ther Assoc. 2014;94(6):875-89. https://doi.org/10.2522/ ptj.20130157.

20. Farah $B Q$, Andrade-Lima A, Germano-Soares AH, Christofaro DGD, Virgı'lio Gomes de Barros M, do Prado WL, et al. Physical activity and heart rate variability in adolescents with abdominal obesity. Pediatr Cardiol. 2018;39(3): 466-72. https://doi.org/10.1007/s00246-017-1775-6.

21. Pradeepa R, Anjana RM, Joshi SR, et al. Prevalence of generalized \& abdominal obesity in urban \& rural. Indian J Med Res. 2015;14:139-50.

22. Khaodhiar L, Cummings S, Apovian CM. Treating diabetes and prediabetes by focusing on obesity management. Curr Diab Rep. 2009;9(5):348-54 https://doi.org/10.1007/s1 1892-009-0055-0.

23. Al Amiri E, Abdullatif $M$, Abdulle $A$, et al. The prevalence, risk factors, and screening measure for prediabetes and diabetes among Emirati overweight/obese children and adolescents. BMC Public Health. 2015. https://doi.org/10.1186/s12889-015-2649-6.

24. Kelishadi R, Growth C. Childhood overweight, obesity, and the metabolic syndrome in developing countries; 2014. https://doi.org/10.1093/epirev/ $\mathrm{mxm003.}$

25. Alam DS, Talukder SH, Chowdhury MAH, et al. Overweight and abdominal obesity as determinants of undiagnosed diabetes and pre-diabetes in Bangladesh. BMC Obes. 2016;3:1-2.

26. Anjana RM, Pradeepa R, Deepa M, Datta M, Sudha V, et al. Prevalence of diabetes and prediabetes ( impaired fasting glucose and / or impaired glucose tolerance ) in urban and rural India : Phase I results of the Indian Council of Medical Research - INdia DIABetes ( ICMR - INDIAB ) study. Diabetologia. 2011;54(12):3022-7. https://doi.org/10.1007/s00125-011-2291-5.

27. Alam DS, Talukder SH, Chowdhury MAH, Siddiquee AT, Ahmed S, Pervin S, et al. Overweight and abdominal obesity as determinants of undiagnosed diabetes and pre-diabetes in Bangladesh. BMC Obes. 2016;3(1):19. https:// doi.org/10.1186/S40608-016-0099-Z.
28. Ganvir RH, Bhalla AK, Dayal D. Growth attainments of Indian children with type 1 diabetes: a mixed longitudinal study. Indian J Pediatr. 2015. https:// doi.org/10.1007/s12098-014-1466-8.

29. Ministry of Health and Family Welfare (MoHFW), Government of India, UNICEF and Population Council. Comprehensive National Nutrition Survey (CNNS) National Report. New Delhi. 2019;1-290.

30. WHO. Use of Glycated Haemoglobin (HbA1c) in the Diagnosis of Diabetes Mellitus Abbreviated Report of a WHO Consultation. 2011.

31. Sherwani SI, Khan HA, Ekhzaimy A, et al. Significance of HbA1c test in diagnosis and prognosis of diabetic patients. Biomarker Insights. 2016. https://doi.org/10.4137/Bmi.s38440.

32. Rani JS. Significance of HbA1C test is a stable indicator of triglycerides in diagnosis and prognosis of diabetic patients. Int J Res Med Sci. 2020. https://doi.org/10.18203/2320-6012.ijrms20204894.

33. McPherson RA, Pincus MR. Chapter 16: Carbohydrates. In: Henry's clinical diagnosis and management by laboratory methods. 22nd ed; 2011. p. 210-25.

34. International Diabetes Federation. Globall IDF/IPAD Guideline for Diabetes in childhood and adolescence; 2011. https://doi.org/10.1097/00007611-1 93212000-00026.

35. Must A, Anderson SE. Body mass index in children and adolescents: Considerations for population-based applications. Int J Obes. 2006;30(4): 590-4. https://doi.org/10.1038/sj.jj.0803300

36. Kumar P, Dhillon P. Length of stay after childbirth in India : a comparative study of public and private health institutions. BMC Pregnancy Childbirth. 2020;20:1-13.

37. Mohan B, Verma A, Singh $K$, et al. Prevalence of sustained hypertension and obesity among urban and rural adolescents : a school-based, crosssectional study in North India. BMJ Open. 2019;9:1-9.

38. Kim HM, Park J, Kim H, et al. Prevalence of the metabolic syndrome in Korean adolescents aged 12-19 years from the Korean National Health and Nutrition Examination Survey 1998 and 2001. Diabetes Res Clin Pract. 2007; 75(1):111-4. https://doi.org/10.1016/j.diabres.2006.04.009.

39. Lambert M, Paradis G, Loughlin JO, et al. PEDIATRIC HIGHLIGHT Insulin resistance syndrome in a representative sample of children and adolescents from Quebec, Canada; 2004. https://doi.org/10.1038/sj.jjo.0802694.

40. Cruz ML, Weigensberg MJ, Huang TT, et al. The metabolic syndrome in overweight Hispanic youth and the role of insulin sensitivity. J Clin Endocrinol Metab. 2004;89(1):108-13. https://doi.org/10.1210/jc.2003-031188.

41. Thomas AS, Greene LF, Ard JD, Oster RA, Darnell BE, Gower BA. Physical activity may facilitate diabetes prevention in adolescents. Diabetes Care. 2009;32(1):9-13. https://doi.org/10.2337/dc08-0780.

42. Lee YS. Consequences of childhood obesity. Ann Acad Med Singap. 2009; 38:75-81.

43. Daniels SR. Complications of obesity in children and adolescents. Int J Obes. 2009;33:S60-5.

44. Gold MA, Gladstein J. Substance use among adolescents with diabetes mellitus: preliminary findings. J Adolesc Heal. 1993;14(2):80-4. https://doi. org/10.1016/1054-139X(93)90089-8.

45. Zamora-Kapoor A, Fyfe-Johnson A, Omidpanah A, et al. Risk factors for prediabetes and diabetes in adolescence and their variability by race and ethnicity. Prev Med (Baltim). 2018. https://doi.org/10.1016/j.ypmed.2018.08. 015.

46. Khan MMA, Karim M, Islam AZ, Islam MR, Khan HTA, Khalilullah MI. Prevalence of overweight and obesity among adolescents in Bangladesh: do eating habits and physical activity have a gender differential effect? J Biosoc Sci. 2019:51(6):843-56. https://doi.org/10.1017/S0021932019000142.

\section{Publisher's Note}

Springer Nature remains neutral with regard to jurisdictional claims in published maps and institutional affiliations. 